\title{
Detection of Low-Flying Target under the Sea Clutter Background Based on Volterra Filter
}

\author{
Hongyan Xing $\mathbb{D}^{1,2}$ and Yan Yan $\mathbb{D}^{1,2}$ \\ ${ }^{1}$ Collaborative Innovation Center on Forecast and Evaluation of Meteorological Disasters, Nanjing University of Information Science \\ \& Technology, Nanjing 210044, China \\ ${ }^{2}$ Jiangsu Key Laboratory of Meteorological Observation and Information Processing, Nanjing University of Information Science \& \\ Technology, Nanjing 210044, China
}

Correspondence should be addressed to Hongyan Xing; xinghy@nuist.edu.cn

Received 1 April 2018; Accepted 13 May 2018; Published 4 July 2018

Academic Editor: Andy Annamalai

Copyright (c) 2018 Hongyan Xing and Yan Yan. This is an open access article distributed under the Creative Commons Attribution License, which permits unrestricted use, distribution, and reproduction in any medium, provided the original work is properly cited.

\begin{abstract}
In order to detect low-flying small targets in complex sea condition effectively, we study the chaotic characteristic of sea clutter, use joint algorithm combined complete ensemble empirical mode decomposition (CEEMD) with wavelet transform to de-noise, and put forward a detection method for low-flying target under the sea clutter background based on Volterra filter. By CEEMD method, sea clutter signal which contains small target can be decomposed into a series of intrinsic mode function (IMF) components, pick out high-frequency components which contain more noise by autocorrelation function, and perform wavelet transform on them. The de-noised components and remaining components are used to reconstruct clear signal. In view of the chaotic characteristics of sea clutter, we use Volterra filter to establish adaptive prediction model, detect low-flying small target hiding in sea clutter background from the prediction error, and compare the root mean square error (RMSE) before and after de-noising to evaluate de-noising effect. Experimental results show that the joint algorithm can effectively remove noise and reduce the RMSE by $40 \%$ at least. Volterra prediction model can directly detect low-flying small target under sea clutter background from the prediction error in the cases of high signal-to-noise ratio (SNR). In the cases of low SNR, after de-noised by joint algorithm, Volterra prediction model can also detect the low-flying small target clearly.
\end{abstract}

\section{Introduction}

The detection of low-flying small targets is the primary problem of low-altitude identification tracking system. The common low-flying small targets mainly contain multirotor unmanned aerial vehicles, tethered balloons, aerial balloons, power delta wings, and so on [1], if enemy uses smart devices such as drones to detect sea condition in our field that may pose a huge security threat [2]. In the current security system, sea surveillance radars are affected by strong sea clutter (waves, weather clutter, etc.). It is quite difficult to "ensure discovery" and "precise position" target at a long distance $[3,4]$. In recent years, it has been found that the inner character of sea clutter can be used to analyze the ocean state and detect small target on sea surface or at low attitude. This is of great theoretical significance and practical value for the safe navigation of ships, civil sea rescue, and military defense construction.

The basic idea of traditional detection method is to assume that sea clutter has characteristics of stationary random process. Scientists adopted two simulation methods of zero memory nonlinear (ZMNL) and spherically invariant random process (SIRP), modeled sea clutter on the basis of stochastic statistical theory. The clutter model was normally Gaussian distribution in the early low-resolution radar, and amplitude distribution of sea clutter obeyed Rayleigh distribution. However, when the radar worked at the small grazing angle, the distribution of sea clutter was significantly deviated from the Gaussian model, and the target detection performance of the Gaussian clutter model dropped sharply. Therefore, the distribution characteristics of sea clutter could be described more accurately by non-Gaussian distribution 
model. The first non-Gaussian model was a logarithmic normal distribution proposed by Trunk and George in 1970 [5]. In 1976, Schleher studied the target detection under Weibull distribution [6]. In the same year, Jakeman and Pusey used the K distribution model to describe sea clutter [7]. In 1980, Sekine et al. analyzed the real sea clutter data of l-band radar and proposed that the amplitude of sea clutter was Log-Weibull distribution [8]. In addition, the Rician distribution was also used to describe the amplitude distribution of sea clutter [9].

With radar, resolution and frequency were getting higher [10]; besides, the distribution of sea clutter was normally influenced by sea surface condition, wind speed, and wave height. It was found that sea clutter did not satisfy the characteristics of linear stochastic stationary processes and the model based on statistical only approximated the probability distribution of sea clutter to some extent and did not reflect the nonlinear dynamic characteristics of sea clutter well. So, it was difficult for traditional methods to make accurate and comprehensive analysis of complex sea clutter. Therefore, the development of nonlinear theory introduced new research methods for the study of sea clutter.

Due to the extreme sensitivity of surface radar echo to initial conditions, scientists began to use chaos theory to study the characteristics of sea clutter. Analyses and researches of chaos time series started at the 1980s. The phase space reconstruction proposed by Kantz and Schreiber was mainstream approach to analyze chaotic time series [11]. According to Takens embedding theorem [12], the selection of embedding dimension and time delay was very important to the finite length chaotic time series, it directly decided whether the reconfiguration phase space has the same topology as the original system. Normally, we use autocorrelation function method [13] and mutual information method [14] to determine the time delay, use GP method [15], FNN method [16], Cao method [17] and so on and to determine embedding dimension.

In 1990, Leung and Haykin et al. applied chaos theory to study sea clutter for the first time [18]. They experimented and observed the correlation dimension of sea clutter time series and found the chaotic characteristics of sea clutter. Subsequently, Haykin et al. analyzed the x-band radar data of McMaster University in Canada firstly and verified the chaotic characteristics of sea clutter by calculating the correlation dimension, Kolmogorov entropy, and Lyapunov exponent $[19,20]$. The famous scientist Vito Volterra proposed the breakthrough concept of Volterra series in the discussion of nonlinear analytic functional; he proved that Volterra series can approach nonlinear systems [21]. Due to the model of Volterra series, both considered effects of linear and nonlinear factors were widely used in nonlinear time series prediction, echo cancellation, and system identification. Despotovic et al. studied a variety of nonlinear Volterra prediction models of chaotic time series [22, 23]. Subsequently, many scholars proposed the application of various algorithms such as least mean square, normalized least mean square, recursive least square, and other algorithms in the identification of Volterra model system [24-27]. Tian and Liu [28] analyzed the sampling signal of ocean echo in time domain of high-frequency radar and verified that it not only has chaotic characteristics but also has fractal characteristics. Jiang et al. [29] of the University of National Defense and Technology studied the chaotic characteristics of the s-band radar sea clutter data and then used the support vector machine to detect the weak targets in the background of sea clutter. Then, Gismero-Menoyo et al. proposed a small target detection method based on radon transform in the background of sea clutter [30].

In terms of signal de-noising, in 2003, Flandrin et al. decomposed the fractal Gaussian noise with empirical mode decomposition method and found that the EMD method can be equivalent to a narrowband filter library to filter the signal [31]. Boudraa and Cexus de-noised signal by filtering and reconstructing the intrinsic mode function (IMF) with different threshold methods, respectively [32]. Kurian and Leung estimated and detected small target signals through reconfigurable dynamic characteristics and chaotic synchronization methods [33]. The RMSE of the experiment result obviously reduced by selecting the appropriate coupling coefficient.

In this paper, we put forward a sea clutter pretreatment method which combined CEEMD and wavelet transform to obtain the clear sea clutter data. By the use of reconstruction phase space technique in chaos theory, one-dimensional sea clutter signal is mapped to high-dimensional space. The phase track of space point is inputted to Volterra adaptive filter. A short-term chaotic sequence prediction model is established. The low-flying small targets under sea clutter background can be detected from the prediction error. The measured sea clutter signals under different conditions are used to verify the effect of the de-noising method combined CEEMD and wavelet transform, test accuracy of Volterra prediction model.

\section{Target Detection Preprocessing Algorithm}

The chaotic characteristics can be used to detect whether the radar echoes of sea clutter contain the low-flying small targets signal. While received sea clutter signal in practical engineering is always influenced by noise, includes the measurement noise inside the radar (quantizing noise and receiver noise) and the dynamic noise from the undulating sea surface. Therefore, de-noising is the primary problem before the study of intrinsic physical properties of sea clutter and detect small target signals.

In the pretreatment of nonlinear non-stationary sea clutter signal, we use joint algorithm combined CEEMD with wavelet transform to de-noise. CEEMD algorithm [34] uses the zero mean characteristic of white noise, improves the EMD algorithm [35] by adding positive and negative relative white noise, solves the problem of modal aliasing and energy leakage occurred when using EMD method to process nonstationary nonlinear signal. Wavelet transform has the specialty of multi-scale, low entropy and decorrelation. It is good at removing random noise and has become a conventional method in signal processing [36]. The multiscale feature of wavelet transform make it has strong local recognition ability. Sea clutter signal can be decomposed into different frequency bands by wavelet transform, each frequency 
band does not overlap, and the frequency bands of decomposition correspond to all frequencies of the original signal.

If only use CEEMD, the high-frequency components are directly filtered, which will lead to the loss of effective information containing in the high-frequency components. The wavelet transform also may filter out useful signal with small amplitude in the process of noise cancellation in wavelet domain. In this paper, we combine CEEMD and wavelet transform to solve above problems. The implementation steps of the joint algorithm are as follows:

(1) Perform wavelet transform on original sea clutter signal to get a suitable threshold.

(2) Adding $n$ groups of auxiliary white noise in positive and negative pairs to the original signal:

$$
\left[\begin{array}{l}
M_{1} \\
M_{2}
\end{array}\right]=\left[\begin{array}{cc}
1 & 1 \\
1 & -1
\end{array}\right]\left[\begin{array}{l}
S \\
N
\end{array}\right] .
$$

In the above equation, $S$ is original sea clutter signal, $N$ is auxiliary noise; and $M_{1}$ and $M_{2}$ are the synthesis signals after adding positive and negative pairs of noise, so the number of signals in the set is $2 n$. Through decomposition of each signal in the set by EMD method, each signal gets a set of IMF components and one residual value, in which the $j$ th IMF component of the $i$ th signal is expressed as $C_{i j}$ and $R$ represents the residual value of each signal, which does not satisfy the conditions of IMF components. The procedure for a standard EMD is detailed in [31].

(3) The decomposition results are obtained by means of multicomponent combinations:

$$
\begin{gathered}
C_{j}=\frac{1}{2 n} \sum_{i=1}^{2 n} C_{i j}, \\
R=\frac{1}{2 n} \sum_{i=1}^{2 n} R_{i} .
\end{gathered}
$$

Among them, $C_{j}$ indicates the final $j$ th IMF component decomposed by CEEMD.

The maximum number of decomposition components is $L$; therefore, the target signal can be expressed as

$$
S=\sum_{j=1}^{L} C_{j}+R .
$$

(4) Calculate the self-correlation function of each IMF component $C_{j}$.

(5) According to the different self-correlation characteristics between noise and signal, select the highfrequency IMF components which mainly including noise: $C_{1} \sim C_{k}$.

(6) Use the threshold obtained in step (1) to perform wavelet transform on high-frequency IMF component which mainly contains noise and get processed IMF components $C_{1}{ }^{\prime} \sim C_{k}{ }^{\prime}$.

(7) Reconstruct signal with the remaining low-frequency IMF components and de-noised IMF components, so we obtain clear sea clutter signal:

$$
S^{\prime}(n)=\sum_{j=1}^{k} C_{j}^{\prime}+\sum_{j=k+1}^{L} C_{j}+R .
$$

\section{Detection of Low-Flying Small Targets in Chaotic Sea Clutter Background}

The traditional low dimensional coordinate system cannot reveal the chaotic characteristics of sea clutter. In order to study its internal complex dynamics better, we map it to high-dimensional space by the phase space reconstruction technique in chaos theory. After the pretreatment of joint algorithm combined CEEMD with wavelet transform, we obtain a pure sea clutter sequence. Considering the chaotic characteristics of sea clutter signal, use CAO algorithm and mutual information method to determine the embedding dimension and delay time of sea clutter chaotic system, respectively, reconstruct phase space [16], use Volterra filter [37] to construct the prediction model, and detect lowflying small targets under the sea clutter background from the prediction error. The Volterra model takes into account both effects of linear and nonlinear factors, tracks the motion trajectory of chaotic sequence adaptively, and accurately predicts many chaotic sequences. Therefore, it is widely used in nonlinear time series prediction, system identification, and interference elimination. In nonlinear prediction model of chaotic time series constructed by Volterra series expansion, input is $X(n)=[x(n), x(n-\tau), \ldots, x(n-(m-1) \tau]$ and output is $\hat{y}(n)=x(n+1)$. The Volterra series expansion of the nonlinear system function is as follows:

$$
\begin{aligned}
\widehat{y}(n) & =h_{0}+\sum_{k=1}^{p} x_{k}(n), \\
x_{k}(n) & =\sum_{i_{1}, \ldots, i_{k}=0}^{m-1} h_{k}\left(i_{1}, \ldots, i_{k}\right) \prod_{j=1}^{k} x\left(n-i_{j} \tau\right) .
\end{aligned}
$$

In the equation above, $h_{k}\left(i_{1}, \ldots, i_{k}\right)$ is called the $k$ order Volterra nucleus and $P$ is the order of Volterra filter. In practical application, this infinite series expansion is difficult to achieve, usually we use finite order truncation and finite summation. In this paper, second order truncated $m$ summation is used in the chaotic time series prediction filter. The second order Volterra model of discrete nonlinear dynamic system is defined as follows:

$$
\begin{aligned}
\widehat{y}(n)= & h_{0}+\sum_{i_{1}=0}^{m-1} h_{1}\left(i_{1}\right) x\left(n-i_{1} \tau\right) \\
& +\sum_{i_{1}, i_{2}=0}^{m-1} h_{2}\left(i_{1}, i_{2}\right) x\left(n-i_{1} \tau\right) x\left(n-i_{2} \tau\right) .
\end{aligned}
$$




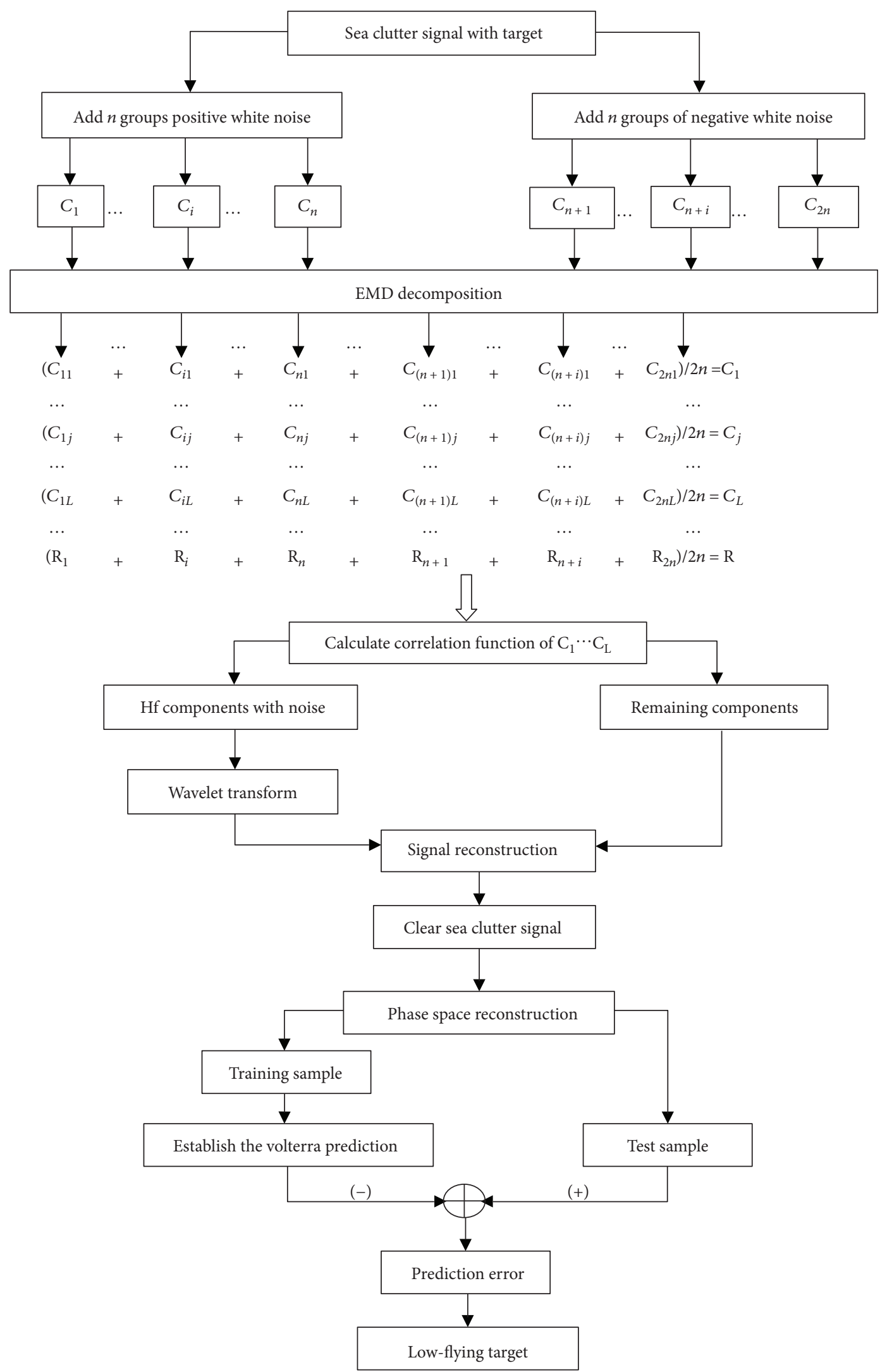

FIGURE 1: Detection process of low-flying target under sea clutter background. 
Among them, $x(\mathrm{n})$ denotes the input sea clutter signal containing low-flying small targets at time $n, \hat{y}(n)$ represents the predicted results obtained by the Volterra adaptive prediction model, and $h_{0}$ is the constant term. $m$ is the memory length of adaptive prediction model and $h_{1}\left(i_{1}\right)$ and $h_{2}\left(i_{1}, i_{2}\right)$ are the first and second order kernel functions, respectively.

The specific process of chaotic prediction of sea clutter based on Volterra adaptive filter are as follows:

(1) Use wavelet transform on original sea clutter signal to get a suitable threshold.

(2) IMF components are obtained by decomposition of CEEMD.

(3) Calculate the self-correlation function of each IMF component, select high-frequency IMF components which mainly contain noise, and use threshold obtained in step (1) to perform wavelet transform de-noising.

(4) Reconstruct signal with remaining low-frequency IMF components and de-noised IMF components so that we can obtain clear sea clutter signal.

(5) The embedding dimension and delay time of sea clutter signal are determined by $\mathrm{CAO}$ algorithm and mutual information method, respectively, using them to reconstruction phase space.

(6) Divide high-dimension point in phase space into training samples and test samples; In all sequences, pick one training sample in every six sampling points and the rest are test samples.

(7) Use selected training samples to establish prediction model based on Volterra filter and adjust the parameters to minimize the prediction error.

(8) Use trained model to predict rest test samples $y(i), i=1,2, \ldots, n$ and get predicted value, comparing the predicted data with the actual data and getting predicted error $\varepsilon(i)=y(i)-\widehat{y}(i)$.

(9) According to different sea clutter characteristics among different sea conditions, determine whether there are low-flying small targets in the prediction error. Root mean square error of prediction error is used to measure the de-noising effect. The formula for RMSE is shown as follows:

$$
E=\sqrt{\frac{\sum_{i=1}^{n} \varepsilon^{2}(i)}{n}} .
$$

The detection flow chart of low-flying small targets in the background of sea clutter is shown in Figure 1.

\section{Experiments and Results}

In order to examine the de-noising performance of joint algorithm combined CEEMD with wavelet transform to sea clutter signal and low-flying small target detection capability
TABLE 1: Main parameters of IPIX radar.

Parameters

Radar transmitting frequency: $9.39 \mathrm{Ghz}$

Polarization mode: HH/VV/HV/VH

Pulse power: $8 \mathrm{~kW}$

Pulse width: $200 \mathrm{~ns}$

Antenna height: $30 \mathrm{~m}$

Antenna gain: $45.7 \mathrm{~dB}$

Antenna diameter: $2.4 \mathrm{~m}$

Antenna type: parabolic

Range resolution: $30 \mathrm{~m}$

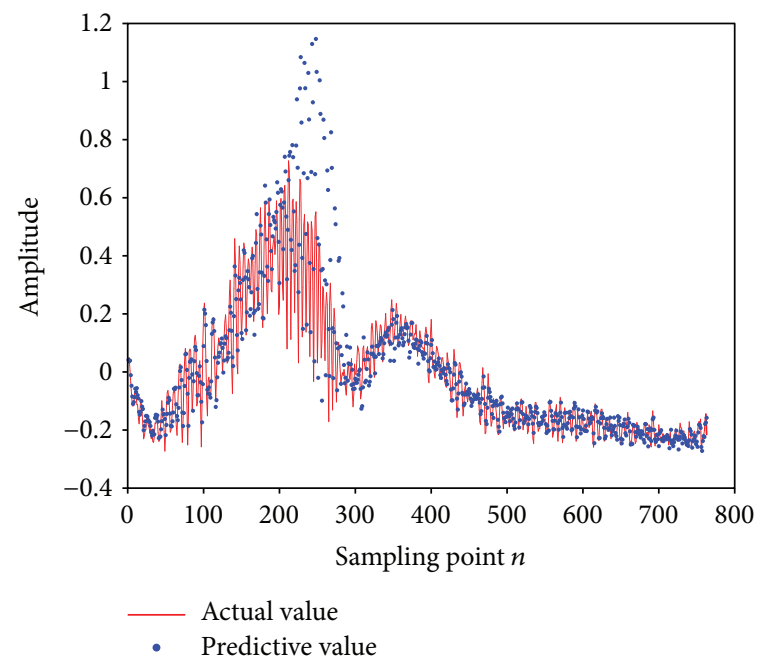

(a)

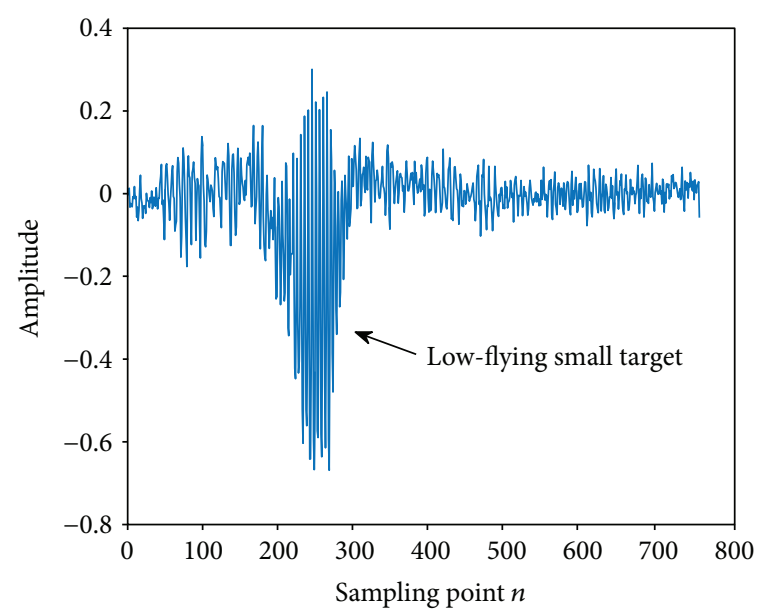

(b)

FIgURE 2: Chaotic prediction of the 280\#8th distance gate sea clutter. (a) Actual value and Volterra predicted value. (b) The prediction error.

of Volterra prediction model under sea clutter background, we use actual sea clutter data for simulation experiments, evaluate the effectiveness of the joint de-noising algorithm by RMSE before and after performance, and observe whether 


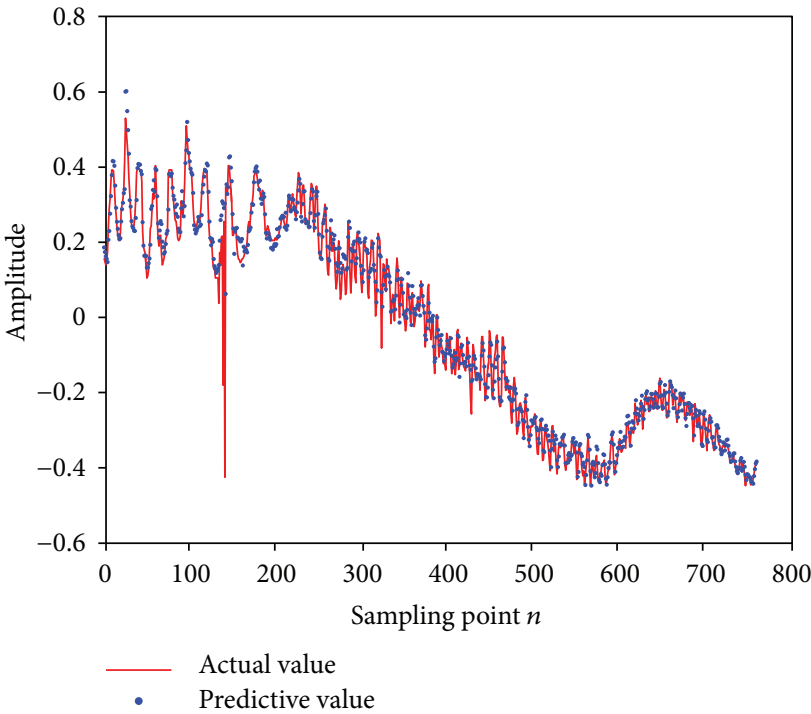

(a)

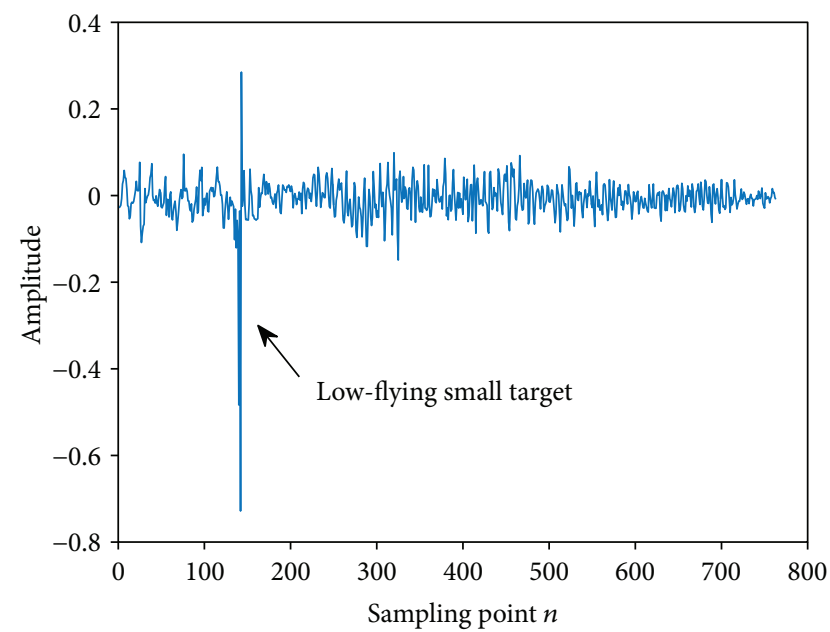

(b)

FIGURE 3: Chaotic prediction of the 320\#7th distance gate sea clutter. (a) Real value and Volterra predicted value. (b) The prediction error.

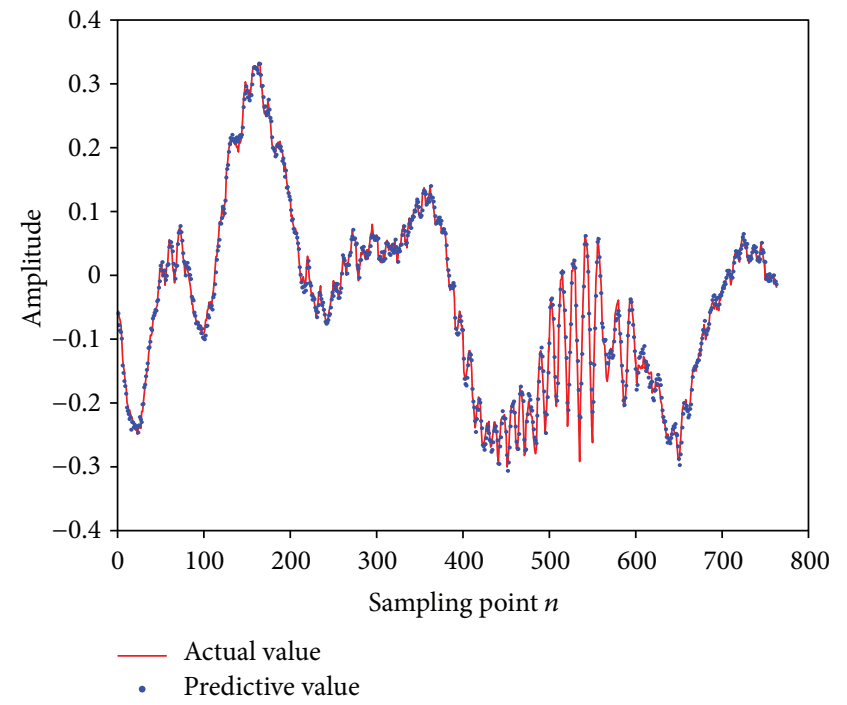

(a)

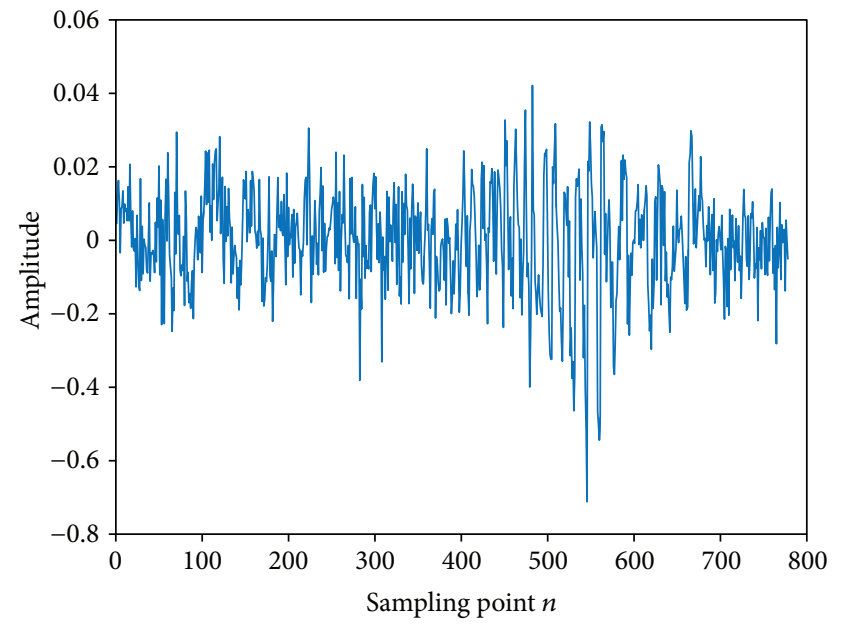

(b)

FIgURE 4: Chaotic prediction of the \#54 8th distance gate sea clutter before de-noising. (a) Real value and Volterra predicted value. (b) The prediction error.

small targets can be clearly identified from the prediction error based on Volterra prediction model.

This paper uses the IPIX radar sea clutter data of McMaster University in Canada. The main parameters of IPIX radar are shown in Table 1.

\subsection{Chaotic Prediction of Sea Clutter Data Based on Volterra} Filter. To test the detection efficiency of chaotic prediction model based on Volterra filter, we choose 1000 sample points of 280\#8th distance gate sea clutter data with the target to experiment. The SNR of this set of data is high. Using CAO algorithm and mutual information method to obtain the embedding dimension which is 8 and the delay time which is 12 , respectively, reconstructs phase space. The points in the phase space are divided into training samples and test samples. In all samples, we choose one in each six samples as training sample and the rest are testing samples and use training samples to build model and high-dimension test samples to predict. The experimental results are shown in Figure 2, and training sampling points are not shown in the figure.

As can be seen from the figure, most of the predicted results agree with the actual value, and the prediction error is small. However, prediction error around 250 points 

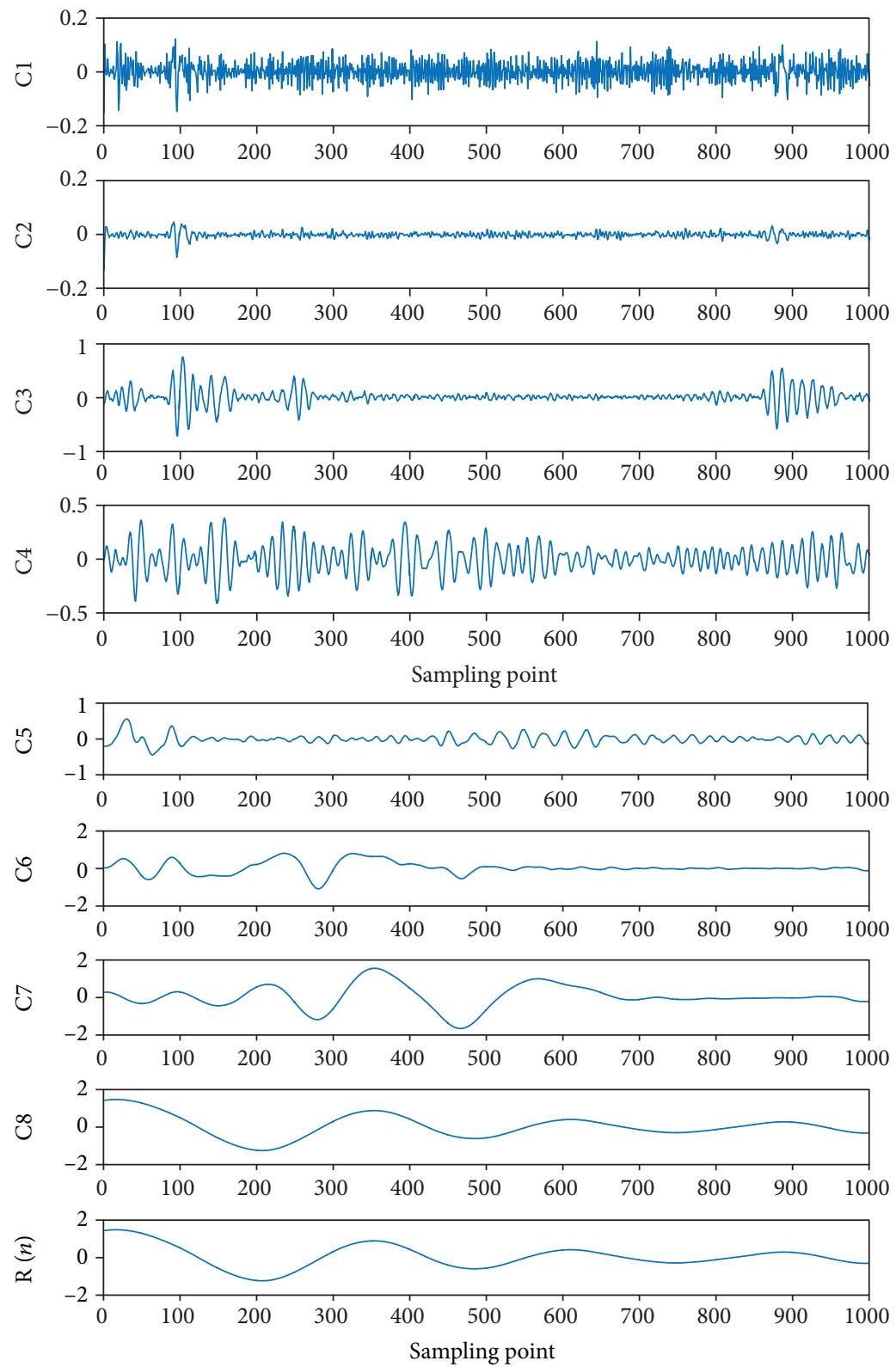

FIgURE 5: Sea clutter decomposition based on CEEMD.

is large, so we can identify that there is exit low-flying target. Experimental result consists of characteristic that the 280\#8th distance gate sea clutter data contains low-flying small goals. To avoid randomness and occasionality, we added a set of experiments and choose 1000 sample points of $320 \# 7$ th distance gate sea clutter data with the target to experiment, results are shown in Figure 3.

It can be clearly seen in the picture that prediction error around 140 points is relatively large. We can infer that there is exit small target and it consists of the characteristic that the $320 \# 7$ th distance gate sea clutter data contains low-flying small goals. Therefore, through the above two groups of experiments, we can verify that the chaotic prediction model based on Volterra filter can effectively detect low-flying small targets under the condition of high SNR.
4.2. Detection of Low-Flying Targets in Sea Clutter under Low SNR Conditions. In practical engineering applications, the processed data generally contain noise, and to solve this problem, we propose a de-noising preprocessing algorithm based on CEEMD and wavelet transform. In order to verify the practicability of the joint algorithm, we choose 1000 sample points of $54 \# 8$ th distance gate sea clutter data which contain target to experiment, using Volterra chaotic prediction model directly. The experimental results are shown in Figure 4.

The root mean square error calculated through (7) is 0.0140 . As can be seen from Figure 4(a), the Volterra chaotic prediction model has good prediction performance. But we cannot identify the target through prediction error clearly. Therefore, performing de-noising pretreatment of joint 

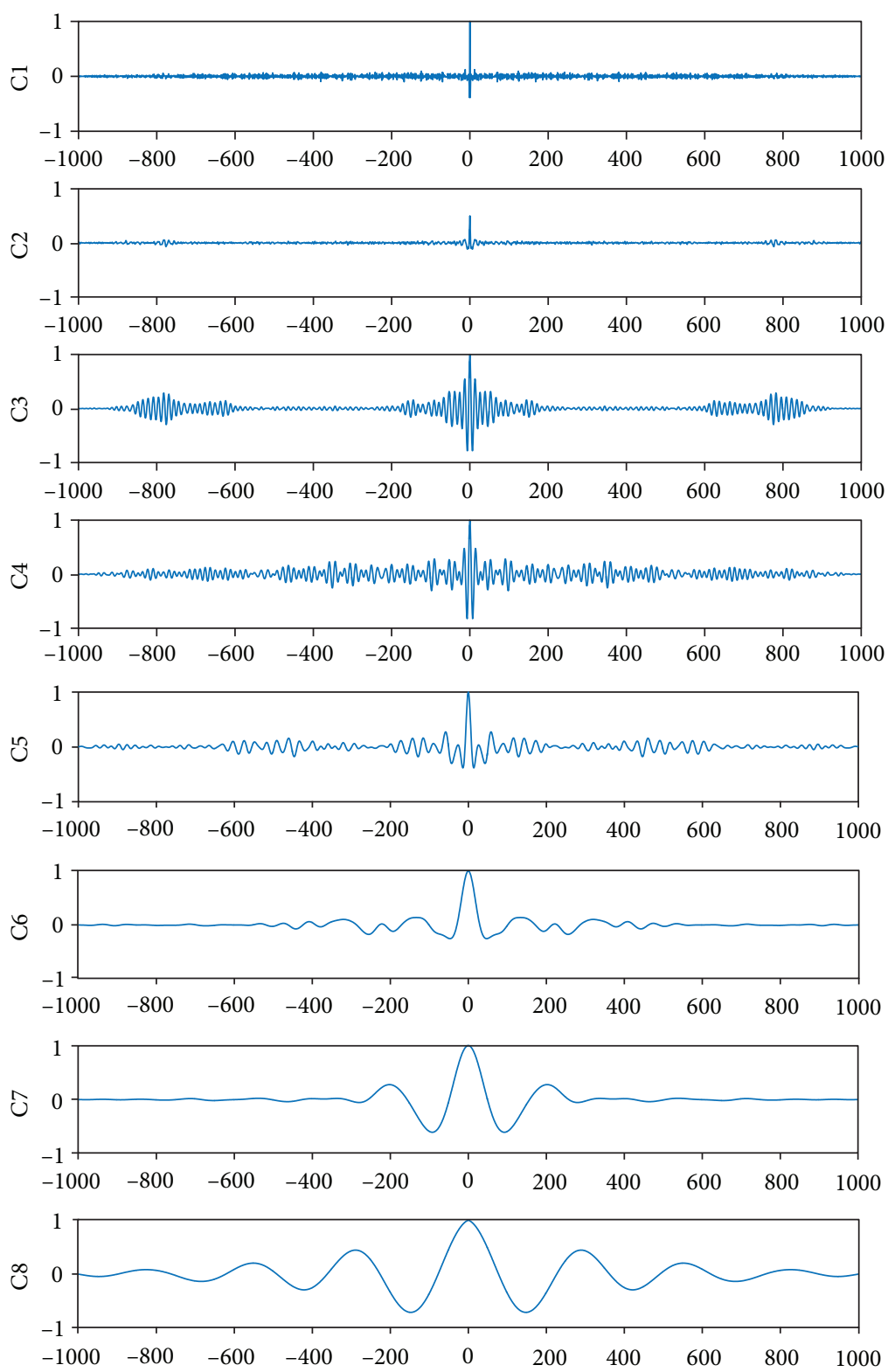

FIgURE 6: Sea clutter decomposition based on CEEMD.

algorithm combined CEEMD with wavelet transform on $54 \# 8$ th distance gate sea clutter data, the specific steps are as follows.

Decompose the sea clutter data by CEEMD so that we can obtain 8 IMF components and residual component $R(n)$ range from high frequency to low frequency, as shown in Figure 5.

Calculating the self-correlation function of 1-9 IMF components, as shown in Figure 6. The useful signal has strong correlation, and the value of its autocorrelation function at nonzero point will change over time. Noise is random and has the characteristics of weak correlation; its value of the autocorrelation function at nonzero point will decay rapidly and approach to 0 . According to the difference between useful signal and noise, we confirm that imf-imf3 components contain a lot of noise.
Then, we perform three-layer wavelet decomposition on imf1-imf3, select the soft threshold de-noising method which may remain more effective information, and use the de-noised IMF components and remaining IMF components to reconstruct clear sea clutter data, as shown in Figure 7.

After the above steps, we obtain the pure sea clutter data. According to the experiment result of 4.1, the embedding dimension is 8 and the delay time is 12 . We reconstruct phase space and send high-dimensional texting points into Volterra prediction model for adaptive prediction. The experimental results are shown in Figure 8.

We compare Figure 4(b) with Figure 8(b) and found that amplitude around the 550 sample points fluctuates greatly; low-flying small target in the background of sea clutter is detected. The RMSE which is obtained by the (7) dropped 

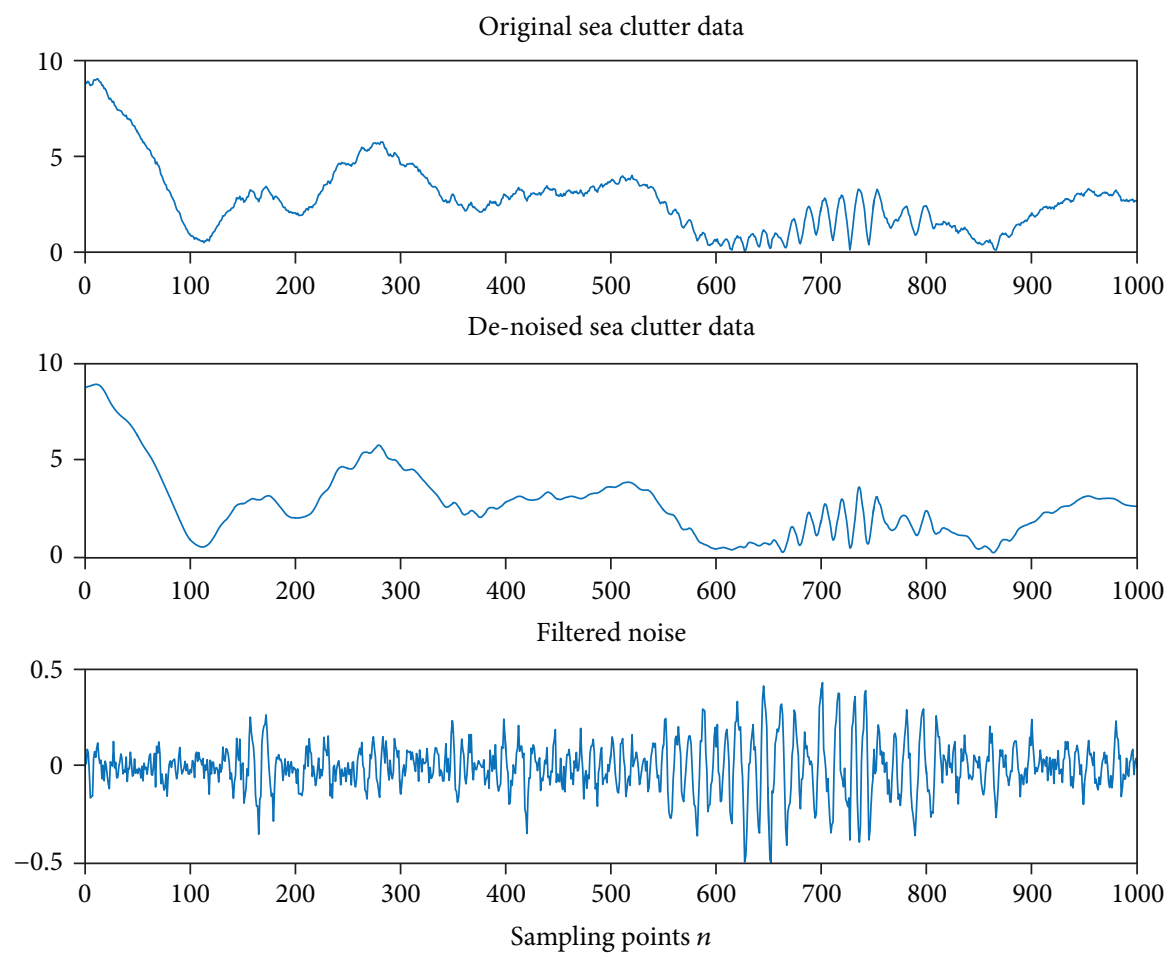

FIGURE 7: De-noising results of sea clutter based on the joint algorithm combined CEEMD with wavelet transform.

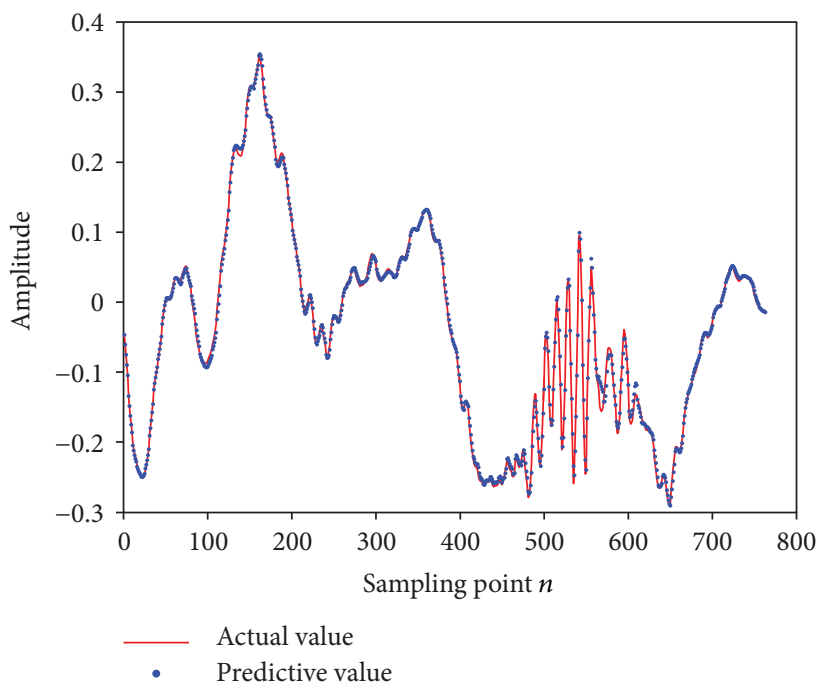

(a)

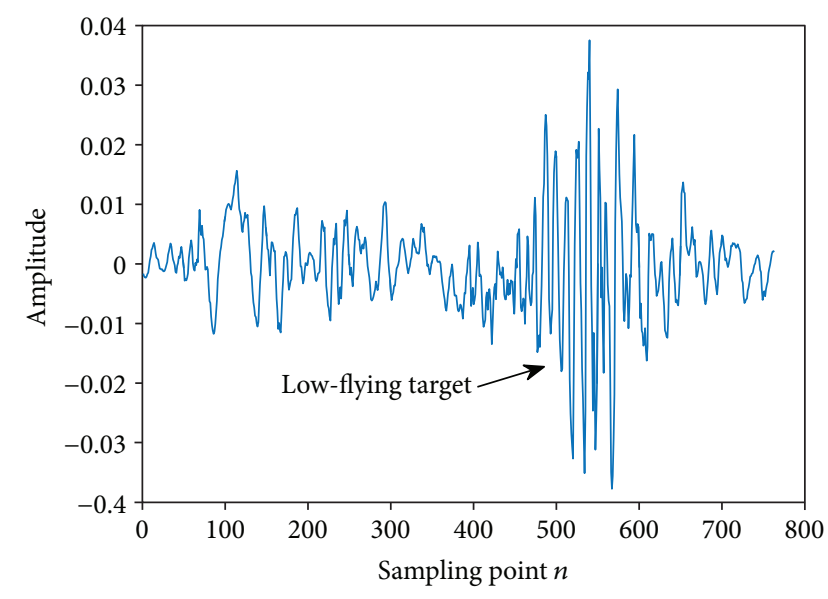

(b)

FIgURE 8: Chaotic prediction of the \#54 8th distance gate sea clutter after de-noising. (a) Real value and Volterra predicted value. (b) The prediction error.

to 0.0084 , compared with the calculated results before de-noising, down by $40 \%$.

To further verify the effectiveness of the algorithm, we use 17\#9th distance gate sea clutter data with the target to experiment. Experimental results are shown in Figure 9(a). Before de-noising, the RMSE calculated by (7) is 0.0569 . As can be seen from the figure, the target cannot be detected from the prediction error, after using joint algorithm combined CEEMD with wavelet transform to de-noise, and the RMSE is 0.0258 , which has decreased by $55 \%$. The prediction error is shown in Figure 9(b). It can be found that there are targets around point 100 and point 600 .

All the above four experiments select the sea clutter data with the target. From the experimental results, it can be seen that the Volterra prediction model can successfully detect low-flying small targets. To avoid the randomness of the 


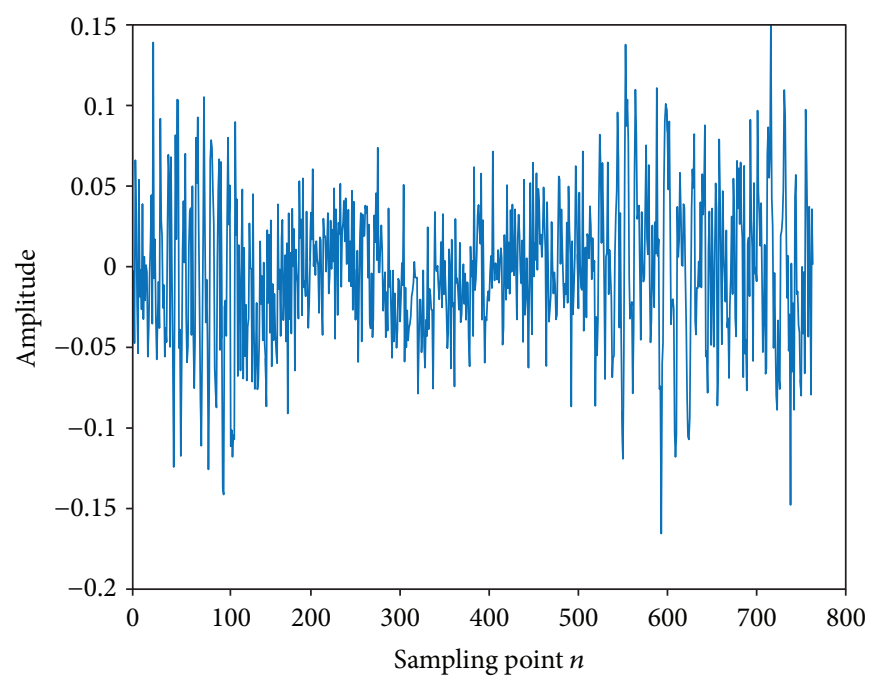

(a)

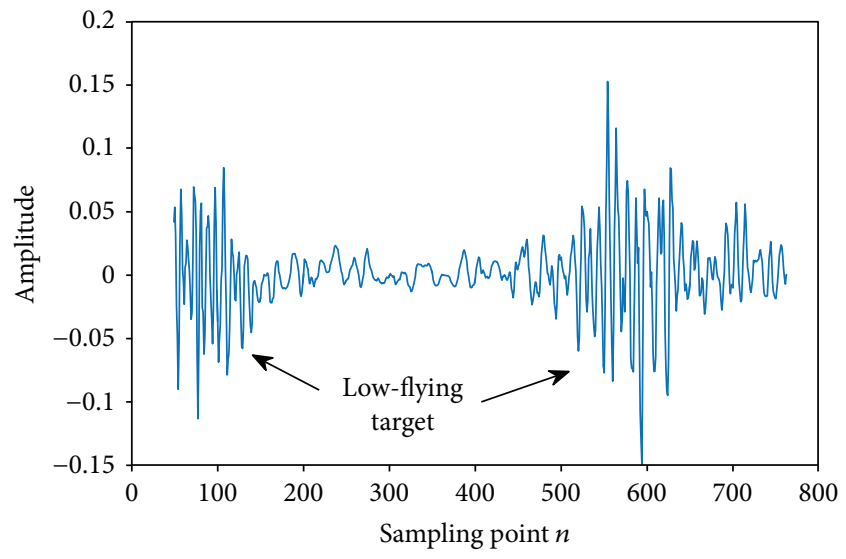

(b)

FIGURE 9: Comparison of prediction error of 17\#9th distance gate sea clutter before and after de-noising. (a) Prediction error before denoising. (b) Prediction error after de-noising.

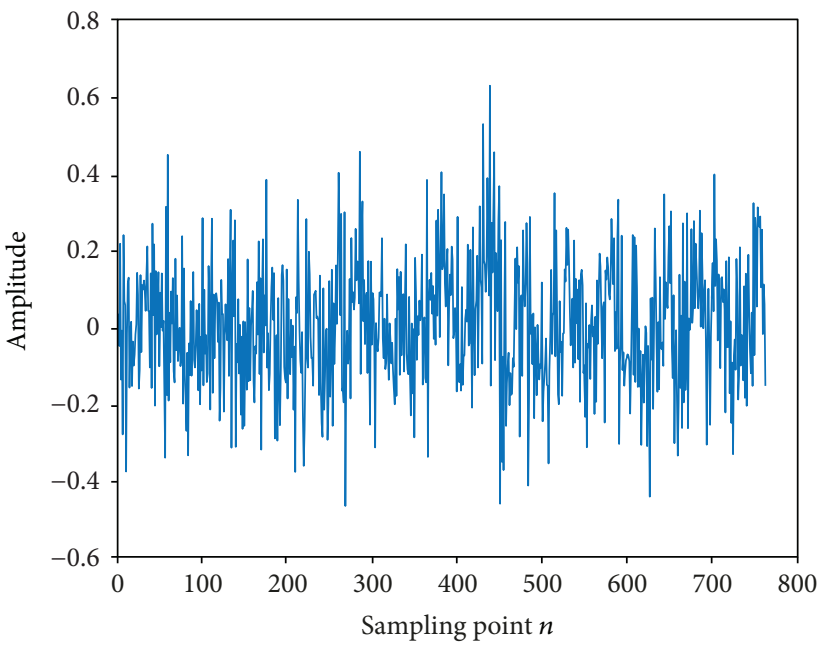

(a)

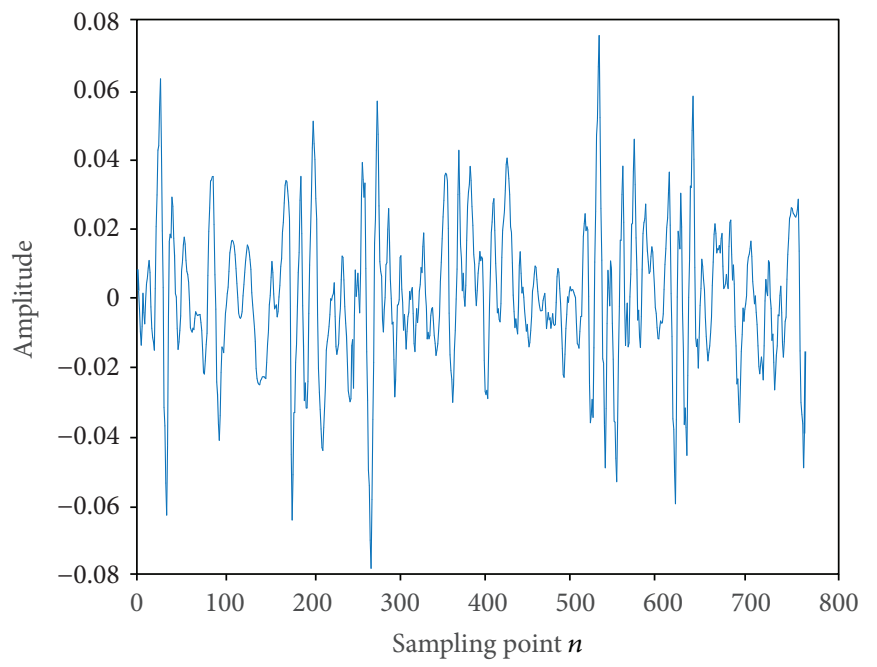

(b)

FIgURE 10: Comparison of prediction error of 17\# 1th distance gate sea clutter before and after de-noising. (a) Prediction error before de-noising. (b) Prediction error after de-noising.

experiment, we selected $17 \# 1$ distance gate sea clutter data for simulation experiment. The experimental results are shown in Figure 10.

Comparing prediction error before and after de-noising, we cannot identify the sampling points with large amplitude fluctuations from the figure, this is consistent with the characteristics of $17 \# 1$ distance gate sea clutter data, which does not contain the target. Therefore, we assure that the Volterra prediction model can actually predict the lowflying small targets in the background of sea clutter.

Through the above simulation experiments, experimental results of $280 \# 8$ th distance sea clutter and $320 \# 7$ th distance sea clutter with target signal show that the proposed Volterra chaotic prediction model based on Volterra adaptive filter can directly detect low-flying small targets signal under high SNR condition effectively.

Under the low SNR condition, we first apply joint algorithm combined CEEMD with wavelet transform to de-noise, then use Volterra prediction model to detect low-flying small targets. The experimental results of $17 \# 9$ distance gate sea clutter data and $54 \# 8$ distance gate sea clutter data show that the proposed joint algorithm can effectively remove the noise while retaining the small target; the RMSE decreased significantly after de-noising. Comparing experiments of $17 \# 9$ distance gate sea clutter data with target and 17\#1 distance sea clutter data without target, results show that the Volterra 
prediction model is effective and can be trusted. Therefore, the pretreatment de-noising method and detection process of low-flying small target in the background of chaos sea clutter background are practical and effective.

\section{Conclusions}

The detection of low-flying small targets in the background of sea clutter is an important branch of radar target detection. It is widely used in both military and civilian applications. In this paper, we study the chaotic characteristics of sea clutter and adaptive characteristic of Volterra filter and propose a low-flying small target detection algorithm based on Volterra filter. In order to effectively remove the interference, we first use the joint algorithm combined CEEMD with wavelet transform to obtain the pure sea clutter signal. It solves the problem that only use CEEMD to de-noise directly and will remove high-frequency components which may contain useful signals.

We process sea clutter signal in chaotic system, project it into high-dimension space to study its internal dynamics, use $\mathrm{CAO}$ algorithm and mutual information method to obtain embedding dimension and delay time of de-noised sea clutter data, and reconstruct phase space. In view of the nonlinear nonstationary characteristics of sea clutter, chaotic time series prediction model based on Volterra adaptive filter is established which can detect low-flying small targets in the background of sea clutter from the prediction error. We use actual sea clutter data to experiment, and the results show that the Volterra prediction model can accurately detect small targets on high SNR conditions directly; however, on low SNR condition, prediction model loses the ability to detect small targets. It is necessary to preprocess the sea clutter data by joint algorithm proposed in this paper combined CEEMD with wavelet transform; this process will reduce the RMSE by at least $40 \%$. After obtaining the pure sea clutter data, Volterra prediction model is used for detection; experiment results show that we can successfully detect the existence of low-flying small targets through prediction error.

In practical engineering applications, sea clutter data are very large. We can first use Volterra prediction model in detecting the low-flying small targets, if results are not clear, use joint algorithm combined CEEMD with wavelet transform to de-noise and, at last, detect low-flying small targets through Volterra prediction model. In next step of research, we try to improve the detection accuracy; more advanced algorithms can be considered to build better mathematical models.

\section{Data Availability}

The raw data required to reproduce these findings are available for download at http://soma.ece.mcmaster.ca/ipix/ dartmouth/. Readers can also write a letter to Simon Haykin (http://www.crl.mcmaster.ca/People/Faculty/Haykin/haykin. html) to obtain data files not listed here.

\section{Conflicts of Interest}

The authors declare that there are no conflicts of interest regarding the publication of this paper.

\section{Acknowledgments}

This work is supported by the National Natural Science Foundation of China (Grant no. 61671248), the Major Program of the National Natural Science Foundation of Jiangsu Province, China (Grant no. 15KJA460008), the Advantage Discipline "Information and Communication Engineering" of Jiangsu Province, China, and the "Summit of the Six Top Talents" Program of Jiangsu Province, China.

\section{References}

[1] X. Zhou, X. Liu, A. Jiang, B. Yan, and C. Yang, "Improving video segmentation by fusing depth cues and the visual background extractor (ViBe) algorithm," Sensors, vol. 17, no. 5, p. 1177, 2017.

[2] J. Pitt, C. Perakslis, and K. Michael, "Drones Humanus [introduction to the special issue]," IEEE Technology and Society Magazine, vol. 33, no. 2, pp. 38-39, 2014.

[3] Z. Goraj, "UAV platforms designed in WUT for border surveillance," in Proceedings of the AIAA Infotech@Aerospace 2007 Conference and Exhibit, Rohnert Park, CA, USA, May 2013.

[4] X. Zhou, X. Liu, C. Yang, A. Jiang, and B. Yan, "Multi-channel features spatio-temporal context learning for visual tracking," IEEE Access, vol. 5, pp. 12856-12864, 2017.

[5] G. V. Trunk and S. F. George, "Detection of targets in nonGaussian sea clutter," IEEE Transactions on Aerospace and Electronic Systems, vol. AES-6, no. 5, pp. 620-628, 1970.

[6] D. Schleher, "Radar detection in Weibull clutter," IEEE Transactions on Aerospace and Electronic Systems, vol. AES-12, no. 6, pp. 736-743, 1976.

[7] E. Jakeman and P. Pusey, "A model for non-Rayleigh sea echo," IEEE Transactions on Antennas and Propagation, vol. 24, no. 6, pp. 806-814, 1976.

[8] M. Sekine, T. Musha, Y. Tomita, T. Hagisawa, T. Irabu, and E. Kiuchi, "Log-Weibull distributed sea clutter," IEE Proceedings F Communications, Radar and Signal Processing, vol. 127, no. 3, pp. 225-228, 1980.

[9] D. M. Drumheller and H. Lew, "Pade approximations to Rician statistical functions," IEEE Transactions on Aerospace and Electronic Systems, vol. 35, no. 4, pp. 1421-1428, 1999.

[10] M. K. Mcdonald, V. Varadan, and H. Leung, "Chaotic behaviour and non-linear prediction of airborne radar sea clutter data," in Proceedings of the Radar Conference, pp. 331-337, Long Beach, CA, USA, April 2001.

[11] H. Kantz and T. Schreiber, Nonlinear Time Series Analysis, Cambridge University Press, 3rd edition, 2004.

[12] F. Takens, "Detecting strange attractors in turbulence," in Dynamical Systems and Turbulence, Warwick 1980, pp. 366381, Springer Berlin Heidelberg, 1981.

[13] B. Hayes, "A progress report on the fine art of turning literature into drivel," Computer Recreations, vol. 249, no. 5, pp. 18-28, 1983. 
[14] A. M. Fraser and H. L. Swinney, "Independent coordinates for strange attractors from mutual information," Physical Review A, vol. 33, no. 2, pp. 1134-1140, 1986.

[15] P. Grassberger and I. Procaccia, "Measuring the strangeness of strange attractors," Physica D: Nonlinear Phenomena, vol. 9, no. 1-2, pp. 189-208, 1983.

[16] M. B. Kennel, R. Brown, and H. D. I. Abarbanel, "Determining embedding dimension for phase-space reconstruction using a geometrical construction," Physical Review A, vol. 45, no. 6, pp. 3403-3411, 1992.

[17] L. Cao, "Practical method for determining the minimum embedding dimension of a scalar time series," Physica D: Nonlinear Phenomena, vol. 110, no. 1-2, pp. 43-50, 1997.

[18] H. Leung and S. Haykin, "Is there a radar clutter attractor?," Applied Physics Letters, vol. 56, no. 6, pp. 593-595, 1990.

[19] S. Haykin and Xiao Bo Li, "Detection of signals in chaos," Proceedings of the IEEE, vol. 83, no. 1, pp. 95-122, 1995.

[20] S. Haykin and S. Puthusserypady, "Chaotic dynamics of sea clutter," Chaos, vol. 7, no. 4, pp. 777-802, 1997.

[21] V. Volterra, Theory of Functionals and Integral and IntegroDifferential Equations, Dover, New York, NY, USA, 1959.

[22] V. Despotovic, N. Goertz, and Z. Peric, "Nonlinear long-term prediction of speech based on truncated Volterra series," IEEE Transactions on Audio, Speech, and Language Processing, vol. 20, no. 3, pp. 1069-1073, 2012.

[23] T. Hélie and B. Laroche, "On the convergence of Volterra series of finite dimensional quadratic MIMO systems," International Journal of Control, vol. 81, no. 3, pp. 358-370, 2008.

[24] J. K. Gruber, D. R. Ramirez, T. Alamo, and C. Bordons, "Nonlinear min-max model predictive control based on Volterra models. Application to a pilot plant," in Proceedings of the IEEE Control Conference, pp. 1112-1117, Budapest, Hungary, August 2009.

[25] Y. Guo, L. Z. Guo, S. A. Billings, D. Coca, and Z. Q. Lang, "Volterra series approximation of a class of nonlinear dynamical systems using the Adomian decomposition method," Nonlinear Dynamics, vol. 74, no. 1-2, pp. 359-371, 2013.

[26] L. M. Li and S. A. Billings, "Analysis of nonlinear oscillators using Volterra series in the frequency domain," Journal of Sound and Vibration, vol. 330, no. 2, pp. 337-355, 2011.

[27] J. J. Jiang, Y. Zhang, and C. Mcgilligan, "Chaos in voice, from modeling to measurement," Journal of Voice, vol. 20, no. 1, pp. 2-17, 2006.

[28] J. S. Tian and T. J. Liu, "Research on chaotic characteristics of HF radar sea clutter," Systems Engineering and Electronics, vol. 29, no. 5, pp. 687-691, 2007.

[29] B. Jiang, H. Q. Wang, X. Li, and G. Gui-Rong, "A novel method of target detection based on the sea clutter," Acta Physica Sinica, vol. 55, no. 8, pp. 3985-3991, 2006.

[30] J. Gismero-Menoyo, A. Asensio-Lopez, A. Asensio-Lopez, and A. Blanco-del-Campo, "Small-target detection in sea clutter based on the radon transform," in Proceedings of the IEEE International Conference on Radar, pp. 610-615, Adelaide, SA, Australia, September 2008.

[31] P. Flandrin, G. Rilling, and P. Goncalves, "Empirical mode decomposition as a filter bank," IEEE Signal Processing Letters, vol. 11, no. 2, pp. 112-114, 2004.

[32] A. O. Boudraa and J. C. Cexus, "EMD-based signal filtering," IEEE Transactions on Instrumentation and Measurement, vol. 56, no. 6, pp. 2196-2202, 2007.
[33] A. P. Kurian and H. Leung, "Weak signal estimation in chaotic clutter using model-based coupled synchronization," IEEE Transactions on Circuits and Systems I: Regular Papers, vol. 56, no. 4, pp. 820-828, 2009.

[34] J. R. Yeh, J. S. Shieh, and N. E. Huang, "Complementary ensemble empirical mode decomposition: a novel noise enhanced data analysis method," Advances in Adaptive Data Analysis, vol. 2, no. 2, pp. 135-156, 2010.

[35] Z. Wu and N. E. Huang, "Ensemble empirical mode decomposition: a noise-assisted data analysis method," Advances in Adaptive Data Analysis, vol. 1, no. 1, pp. 1-41, 2009.

[36] A. C. To, J. R. Moore, and S. D. Glaser, "Wavelet denoising techniques with applications to experimental geophysical data," Signal Processing, vol. 89, no. 2, pp. 144-160, 2009.

[37] J. Zhang and Y. Pang, "Pipelined robust M-estimate adaptive second-order Volterra filter against impulsive noise," Digital Signal Processing, vol. 26, no. 26, pp. 71-80, 2014. 


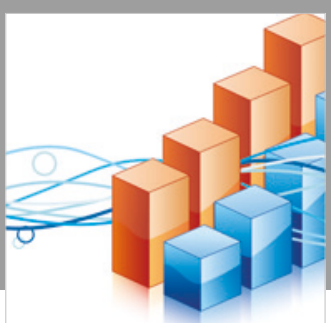

Advances in

Operations Research

\section{-n-m}
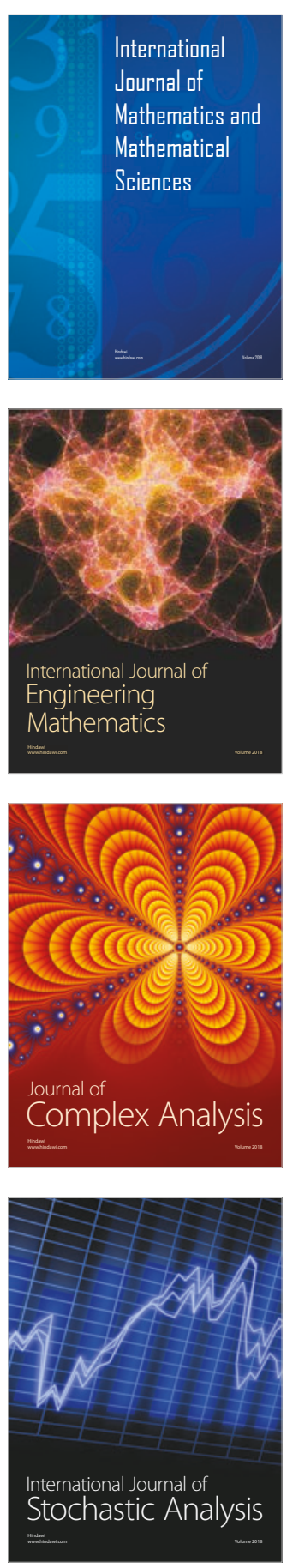
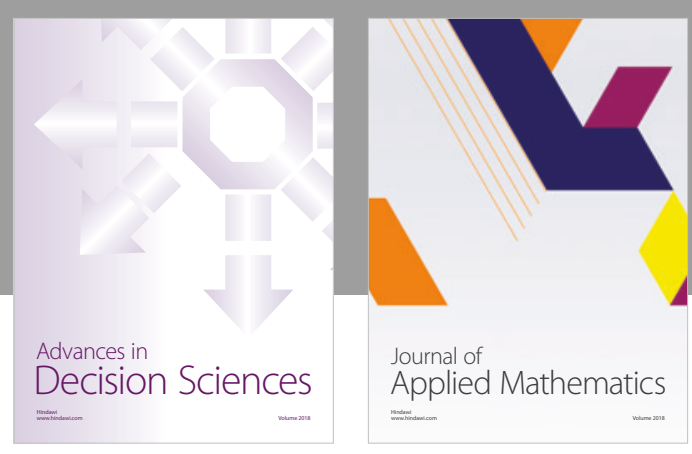

Journal of

Applied Mathematics
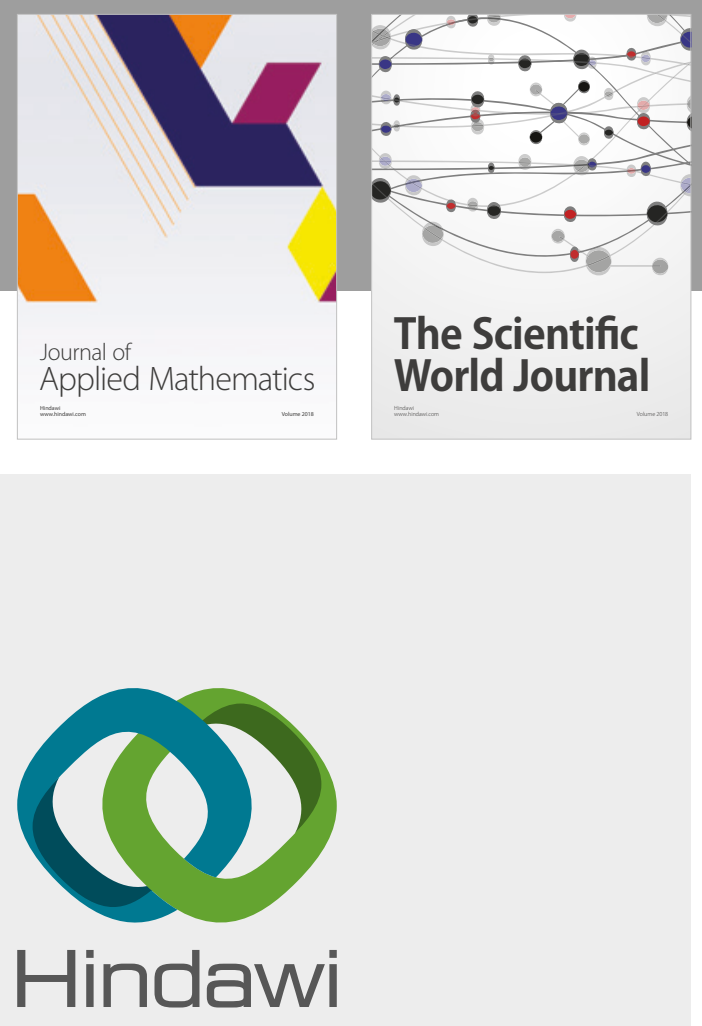

Submit your manuscripts at

www.hindawi.com

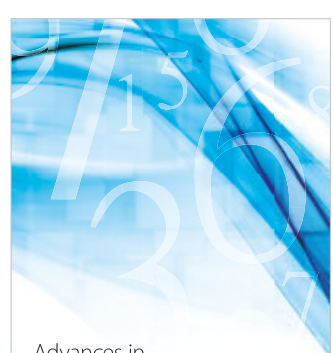

Advances in
Numerical Analysis
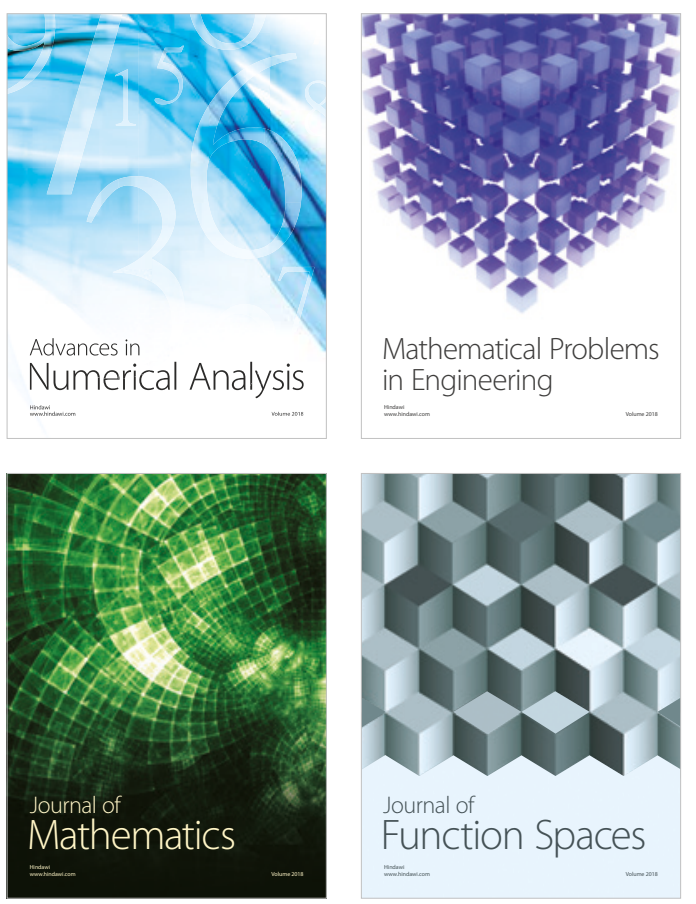

Mathematical Problems in Engineering

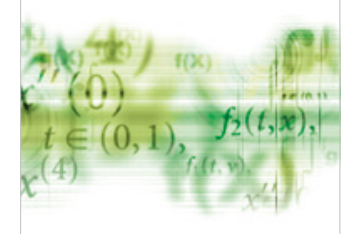

International Journal of

Differential Equations

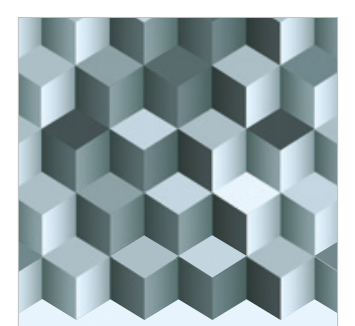

Journal of

Function Spaces
The Scientific

World Journal

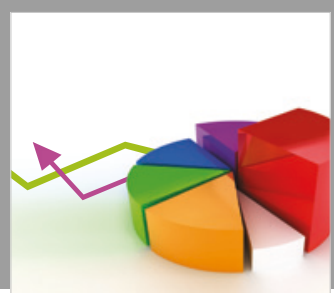

Journal of

Probability and Statistics
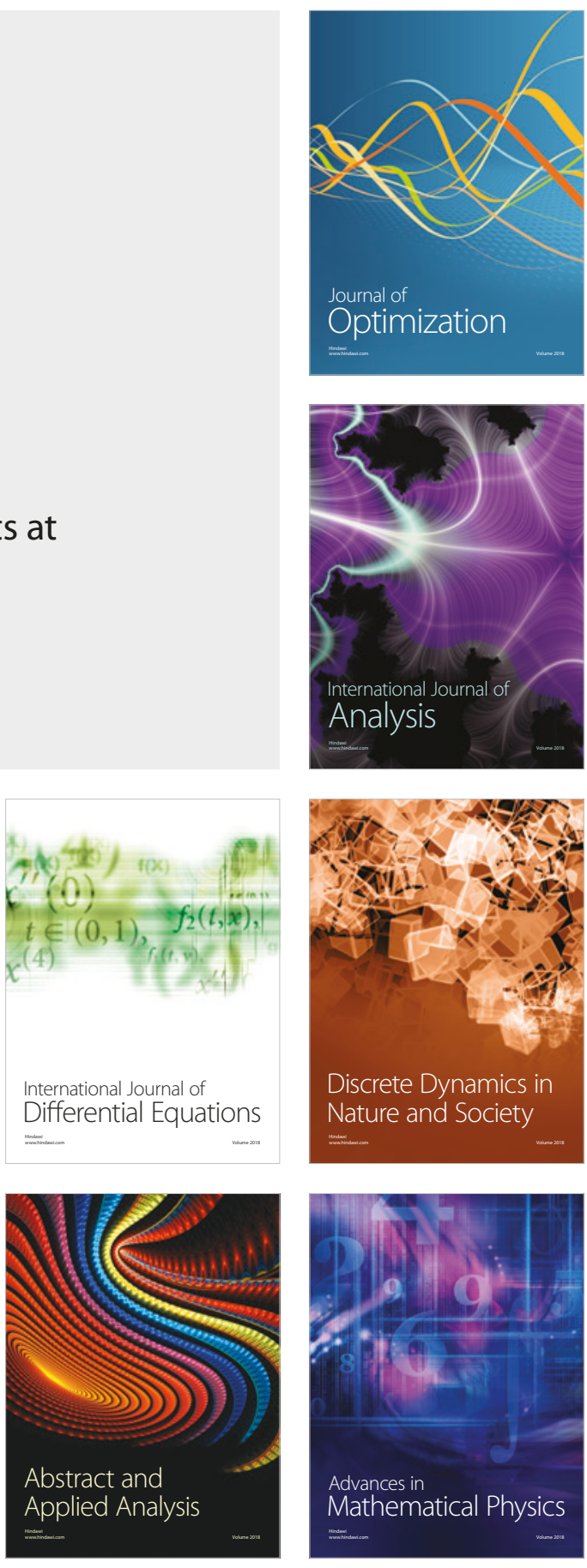